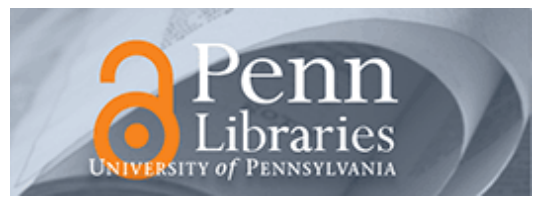

University of Pennsylvania

ScholarlyCommons

Statistics Papers

Wharton Faculty Research

$12-1984$

\title{
Diffusion, Cell Mobility, and Bandlimited Functions
}

\author{
H. J. Landau \\ B. F. Logan \\ Larry A. Shepp \\ University of Pennsylvania
}

N. Bauman

Follow this and additional works at: https://repository.upenn.edu/statistics_papers

Part of the Applied Mathematics Commons, and the Statistics and Probability Commons

\section{Recommended Citation}

Landau, H. J., Logan, B. F., Shepp, L. A., \& Bauman, N. (1984). Diffusion, Cell Mobility, and Bandlimited Functions. SIAM Journal of Applied Mathematics, 44 (6), 1232-1245. Retrieved from https://repository.upenn.edu/statistics_papers/412

This paper is posted at ScholarlyCommons. https://repository.upenn.edu/statistics_papers/412

For more information, please contact repository@pobox.upenn.edu. 


\title{
Diffusion, Cell Mobility, and Bandlimited Functions
}

\begin{abstract}
The mechanism by which leukocytes steer toward a chemical attractant is not fully resolved. Experimental data suggest that these cells detect differences in concentration of chemoattractant over their surface and "walk" up the gradient. The problem has been considered theoretically only in stationary media, where the distribution of attractant is determined solely by diffusion. Experimentally, bulk flow has been allowed only unintentionally. Since bulk flow is characteristic of real systems, we examine a simple two-dimensional model incorporating both diffusion and an additional drift. The latter problem leads to an integral equation which is central also in the study of weighted Hilbert spaces of bandlimited functions. We find asymptotic expressions for the required solution by a Wiener-Hopf method adapted to a finite interval. We conclude that, without drift, the concentration does not vary detectably around the cell, but that drift inceases this variation substantially. Thus over model suggests that drift may play an important role in the cell's chemotactic response.

Disciplines

Applied Mathematics | Statistics and Probability
\end{abstract}


Diffusion, Cell Mobility, and Bandlimited Functions

Author(s): H. J. Landau, B. F. Logan, L. A. Shepp, N. Bauman

Source: SIAM Journal on Applied Mathematics, Vol. 44, No. 6 (Dec., 1984), pp. 1232-1245

Published by: Society for Industrial and Applied Mathematics

Stable URL: http://www.jstor.org/stable/2101408

Accessed: 22/11/2010 14:07

Your use of the JSTOR archive indicates your acceptance of JSTOR's Terms and Conditions of Use, available at http://www.jstor.org/page/info/about/policies/terms.jsp. JSTOR's Terms and Conditions of Use provides, in part, that unless you have obtained prior permission, you may not download an entire issue of a journal or multiple copies of articles, and you may use content in the JSTOR archive only for your personal, non-commercial use.

Please contact the publisher regarding any further use of this work. Publisher contact information may be obtained at http://www.jstor.org/action/showPublisher?publisherCode=siam.

Each copy of any part of a JSTOR transmission must contain the same copyright notice that appears on the screen or printed page of such transmission.

JSTOR is a not-for-profit service that helps scholars, researchers, and students discover, use, and build upon a wide range of content in a trusted digital archive. We use information technology and tools to increase productivity and facilitate new forms of scholarship. For more information about JSTOR, please contact support@jstor.org. 


\title{
DIFFUSION, CELL MOBILITY, AND BANDLIMITED FUNCTIONS*
}

\author{
H. J. LANDAU $\dagger$, B. F. LOGAN $\dagger$, L. A. SHEPP $\dagger$ AND N. BAUMAN $¥$
}

\begin{abstract}
The mechanism by which leukocytes steer toward a chemical attractant is not fully resolved. Experimental data suggest that these cells detect differences in concentration of chemoattractant over their surface and "walk" up the gradient. The problem has been considered theoretically only in stationary media, where the distribution of attractant is determined solely by diffusion. Experimentally, bulk flow has been allowed only unintentionally. Since bulk flow is characteristic of real systems, we examine a simple two-dimensional model incorporating both diffusion and an additional drift. The latter problem leads to an integral equation which is central also in the study of weighted Hilbert spaces of bandlimited functions. We find asymptotic expressions for the required solution by a Wiener-Hopf method adapted to a finite interval. We conclude that, without drift, the concentration does not vary detectably around the cell, but that drift inceases this variation substantially. Thus over model suggests that drift may play an important role in the cell's chemotactic response.
\end{abstract}

1. Introduction. The mechanism by which cells steer toward a chemical attractor is still something of a mystery, for both bacteria and cells of higher organisms succeed at this task even when the concentration of the chemoattractant is so low that statistical fluctuations alone would seem to preclude accurate assessment. Bacteria, which can swim rapidly through the mediurn, apparently overcome this difficulty by striking out in a random direction, traversing a sizable distance, and then choosing a new random direction if the concentration has fallen [8]. Mammalian leukocytes, on the other hand, move very slowly along surfaces by directed extension of pseudopods; they are claimed to detect spatial gradients, and to "walk" up the gradient [9]. The plausibility of such an explanation, however, depends on how accurately the cell can measure a concentration, and on whether the concentration varies sufficiently around the cell for a gradient to be detectable. These questions were addressed in [1], for the case that molecules of the chemoattractant propagate only by diffusion. Body fluids, however, are in constant motion. We therefore analyze here a simple two-dimensional model of a stationary cell in a fluid which moves, and so imparts an additional drift to the diffusing chemoattractant. This problem leads to an integral equation of finite convolution type which, surprisingly, is central also in a very different context: that of weighted Hilbert spaces of bandlimited functions. We prove existence of a solution for a class of such equations and, specializing to the one at hand, apply a WienerHopf method to obtain asymptotic expressions for the required variation. We conclude that drift may play an important role in the cell's chemotactic response.

2. The model and results. In coordinates relative to the cell, we view the cell as a fixed vertical segment $I_{L}:|y| \leqslant L$ of the $y$-axis, and the attractor as a number of particles, each independently executing a two-dimensional Brownian motion $z=z_{t}$ with horizontal drift $c \geqslant 0$, starting at a point $x$ far away on the negative $x$-axis.

*Received by the editors June 14, 1983 and in revised form April 27, 1984. This paper was typeset by Gisele Wallace and Mary E. McGrath at AT\&T Bell Laboratories, Murray Hill, New Jersey, using the troff program running under the Unix ${ }^{\text {TM }}$ operating system.

†AT\&T Bell Laboratories, Murray Hill, New Jersey 07974.

$¥$ Medical Research Division, American Cyanamid, Lederle Laboratories, Pearl River, New York 10965 
We are interested in the probability that the motion first hits $I_{L}$ from the right, or distant, side, thereby conveying false information as to the location of the source. Thus let $\tau$ be the first time that $z$ hits $I_{L}$ (setting $\tau=\infty$ if $z$ never hits $I_{L}$ ), let

$$
Q(c, L, x)=\operatorname{Prob}(\tau<\infty),
$$

and let $R=R(c, L, x)$ denote the conditional probability that $z$ hits $I_{L}$ from the right, given that $\tau<\infty$ :

$$
R(c, L, x)=\operatorname{Prob}\left(z_{\tau-}=0+\mid \tau<\infty\right) .
$$

Then $(1-R) / R$ represents the ratio of concentrations of the attractor on opposite sides of the cell; the closer it is to 1 , the more difficult to distinguish the direction of the source.

When there is no drift, the motion always hits $I_{L}$, and a simple construction determines $R(0, L, x)$ explicitly:

$$
\begin{aligned}
& Q(0, L, x) \equiv 1, \\
& R(0, L, x)=1 / 2-\frac{1}{\pi} \tan ^{-1} \frac{L}{|x|} .
\end{aligned}
$$

For $c>0$, however, the problem is more difficult and leads to the measure $\mu_{0}$ satisfying the integral equation

$$
\int_{-T}^{T} K_{0}(t-s) d \mu_{0}(s)=1, \quad|t| \leqslant T,
$$

where $K_{0}$ is the Bessel function

$$
K_{0}(t)=\int_{0}^{\infty} \frac{\cos u t}{\sqrt{1+u^{2}}} d u,
$$

and $T=L c$. (Here $t$ is a dimensionless variable, not time, while $c$ is the drift velocity scaled by twice the diffusion coefficient; thus in physical units $T=L c / 2 D$, and is likewise dimensionless.) Curiously, this equation arises also in studying the Hilbert space of functions of exponential type $T$, in which the scalar product is computed using the weight $1 / \sqrt{1+t^{2}}$; for that reason, it has appeared in the work of several authors, notably M. G. Krein [5,][6]. We show first by construction that (5) has a unique solution whenever its kernel is even, nonnegative, convex decreasing, and integrable. An independent Hilbert space argument, applicable to a larger class of kernels, is also available. We then derive the following description of the behavior of $Q$ and $R$ when $x \rightarrow-\infty$;

$$
\begin{gathered}
Q(c, L, x) \sim \sqrt{\frac{\pi}{2 c|x|} \mu_{0}[-T, T]}, \\
R(c, L, x) \rightarrow \frac{1}{2}-\frac{T}{\pi \mu_{0}[-T, T]}
\end{gathered}
$$

For the quantity of interest $\mu_{0}[-T, T]$, we obtain

$$
\pi \mu_{0}[-T, T]=1+2 T+o\left(e^{-2 T}\right), \text { as } T \rightarrow \infty,
$$

and, for small $T$, 


$$
\mu_{0}[-T, T] \sim \frac{1}{\log \frac{1}{T}} .
$$

By a broad theory [7] recently developed for equations such as (5), (9) can be replaced by the more precise bound

$$
1+2 T-\frac{e^{-2 T}}{\sqrt{\pi}(2 T)^{3 / 2}} \leqslant \pi \mu_{0}[-T, T] \leqslant 1+2 T,
$$

but we will not prove this here.

To apply these results, we observe first that without drift, by (4), the discrimination ratio $(1-R) / R$ for large $|x| / L$ behaves as $1+(4 / \pi) \tan ^{-1}(L /|x|)$, and directionality is quickly lost even for only moderate displacement of the cell from the source. On the other hand, taking cell size to be approximately 10 microns, so that $L \sim 5 \times 10^{-4} \mathrm{~cm}, \quad D \sim 10^{-6} \mathrm{~cm}^{2} / \mathrm{sec}$ for proteins, and $c \sim 4$ cell diameter $/ \mathrm{sec}$ $\sim 4 \times 10^{-3} \mathrm{~cm} / \mathrm{sec}$, yields $T=L c / 2 D \sim 1$, and by (8) and (9)

$$
(1-R) / R \sim 5,
$$

considerably magnifying the directionality. For $T=1 / 2$, the ratio is approximately 3 , and even for $T=1 / 4,(10)$ gives $(1-R) / R \sim 1.6$. We conclude that, without drift, the concentration does not vary detectably around the cell, but that drift increases this variation substantially. Thus our model suggests that drift may play an important role in the cell's steering mechanism.

3. Proofs. Let $z_{t}(c, x, y)$ be a Brownian motion in two dimensions with drift $c \geqslant 0$ in the $x$-direction, starting from a point $(x, y)$ :

$$
z_{t}(c, x, y)=\left(W_{t}^{(1)}+c t+x, W_{t}^{(2)}+y\right), \quad t \geqslant 0,
$$

where $W_{t}^{(1)}$ and $W_{t}^{(2)}$ are independent Wiener processes. Let $\tau$ be the first time to hit the segment $I_{L}=\{x=0,|y| \leqslant L\}$, with $\tau=\infty$ if $I_{L}$ is never hit, and let $f$ be the function defined on the boundary of the plane slit along $I_{L}$ by

$$
\begin{aligned}
& f(0-, y)=1, \\
& f(0+, y)=i, \quad|y| \leqslant L .
\end{aligned}
$$

This choice of values will make it possible to compute simultaneously the probabilities of hitting $I_{L}$ from either side. Introducing the random variable $f\left[z_{\tau}(c, x, y)\right]$ and setting

$$
u(x, y)=E\left\{f\left[z_{\tau}(c, x, y)\right]\right\}
$$

we see from the definition of $f$ that the real and imaginary parts of $u$ represent the probabilities that $z(c, x, y)$ first hits $I_{L}$ from the left and right, respectively. The function $u$ is now defined and bounded in the slit plane, and by properties of Brownian motion, $u$ is continuous, with boundary values 1 and $i$, respectively, on the left and right sides of the slit. Moreover [3, pp. 1-3], $u$ satisfies the differential equation

$$
2 c u_{x}+\left(u_{x x}+u_{y y}\right)=0, \quad(x, y) \notin I_{L},
$$

with the boundary condition 


$$
u(x, y)=f(x, y), \quad(x, y) \in I_{L} .
$$

In this equation, the diffusion coefficient $D$ has been normalized to $1 / 2$; thus in physical units, $c$ should be replaced by $c / 2 D$, a quantity having dimension (length) ${ }^{-1}$.

When there is no drift, (11) shows that $u$ is harmonic, and so can be found by a linear transformation from the angle subtended at $(x, y)$ by $I_{L}$, which has values $\pm \pi$ on the two sides of $I_{L}$. Specifically,

$$
u(x, y)=\frac{1+i}{2}-\frac{1-i}{2 \pi} \arg \left(\frac{\zeta+i L}{\zeta-i L}\right), \quad \zeta=x+i y .
$$

Since $Q(0, L, x)=\operatorname{Re} u(x, 0)+\operatorname{Im} u(x, 0)$ while $R(0, L, x)=\operatorname{Im} u(x, 0)$, (3) and (4) follow immediately.

For $c>0$, we seek a solution to (11) as a superposition of exponentials $e^{s x-i r y}$; here, $s$ and $r$ have dimension (length) ${ }^{-1}$, so that the exponent is dimensionless. To satisfy (11) we require

$$
2 c s+\left(s^{2}-r^{2}\right)=0
$$

or

$$
s=-c \pm \sqrt{c^{2}+r^{2}}
$$

We now write

$$
u(x, y)=\left\{\begin{array}{l}
\frac{1}{\sqrt{2 \pi}} \int_{-\infty}^{\infty} a(r) e^{\left(-c+\sqrt{c^{2}+r^{2}}\right) x} e^{-i r y} d r, \quad x<0, \\
\frac{1}{\sqrt{2 \pi}} \int_{-\infty}^{\infty} b(r) e^{\left(-c-\sqrt{c^{2}+r^{2}}\right) x} e^{-i r y} d r, \quad x>0,
\end{array}\right.
$$

the different exponential factors being chosen so as to ensure boundedness of $u ; a(t)$ and $b(t)$ have dimension of (length). (For symmetry, we will throughout use the Fourier and inverse Fourier transforms with the factor $1 / \sqrt{2 \pi}$.) Since $u(x, y)$ and $u_{x}(x, y)$ are continuous across $x=0,|y|>L$, we require

$$
\begin{gathered}
\int_{-\infty}^{\infty}[a(r)-b(r)] e^{-i r y} d r=0,|y|>L, \\
\int_{-\infty}^{\infty}\left\{-c[a(r)-b(r)]+\sqrt{c^{2}+r^{2}}[a(r)+b(r)]\right\} e^{-i r y} d r=0,|y|>L,
\end{gathered}
$$

so that

$$
\int_{-\infty}^{\infty} \sqrt{c^{2}+r^{2}}[a(r)+b(r)] e^{-i r y} d r=0,|y|>L .
$$

Moreover, from the boundary conditions on $u(x, y)$,

$$
\begin{aligned}
& \frac{1}{\sqrt{2 \pi}} \int_{-\infty}^{\infty} a(r) e^{-i r y} d r=1, \quad|y| \leqslant L, \\
& \frac{1}{\sqrt{2 \pi}} \int_{-\infty}^{\infty} b(r) e^{-i r y} d r=i, \quad|y| \leqslant L .
\end{aligned}
$$


Taken together, (15), (16), and (13) show that

$$
a(r)-b(r)=(1-i) \frac{2 \sin L r}{\sqrt{2 \pi} r}
$$

while

$$
\frac{1}{\sqrt{2 \pi}} \int_{-\infty}^{\infty}[a(r)+b(r)] e^{-i r y} d r=(1+i), \quad|y| \leqslant L .
$$

Now (14) and (18) can be viewed as conditions to determine $a$ and $b$. Putting these in more convenient rescaled form, we see that our problem has been reduced to that of solving

$$
\begin{gathered}
\int_{-\infty}^{\infty} \sqrt{1+t^{2}} p(t) e^{-i y t} d t=0, \quad|y|>T, \\
\int_{-\infty}^{\infty} p(t) e^{-i y t} d t=1, \quad|y| \leqslant T,
\end{gathered}
$$

with $T=L c$, for on setting

$$
a(r)+b(r)=p\left(\frac{r}{c}\right) \frac{\sqrt{2 \pi}(1+i)}{c}
$$

we satisfy (14) and (18); we note also that this rescaling by $c$ makes the variables $t$ and $T$ of (19) dimensionless. Then, combining (21) with (17) we can determine $a$ and $b$ separately, hence also $u(x, y)$. We should note that, in (14), the integrand is not necessarily required to be integrable; rather, the integral is, by its derivation, interpreted as the result of a limiting process. Thus we view (19) also as asserting only that, in a decomposition of $\sqrt{1+t^{2}} p(t)$ into exponentials, only frequencies $|y| \leqslant T$ appear, or, equivalently, that $\sqrt{1+t^{2}} p(t)$ is extendable from the reals as an entire function of exponential type no greater than $T$.

We now proceed to show, by two distinct approaches, that equations (19) and (20) have a solution. The first of these is constructive; the second, abstract but more general. To start, suppose that $\sqrt{2 / \pi} \sqrt{1+t^{2}} p(t)$ is the (inverse) Fourier transform of some bounded measure $d \mu$ which, by (19), is necessarily supported in $|y| \leqslant T$.

$$
\frac{2}{\sqrt{2 \pi}} \sqrt{1+t^{2}} p(t)=\frac{1}{\sqrt{2 \pi}} \int_{-T}^{T} e^{i s t} d \mu(s) \text {. }
$$

Then on writing

$$
\begin{aligned}
\sqrt{2 \pi} p(t) & =\sqrt{2 \pi} \frac{2}{\sqrt{2 \pi}} \sqrt{1+t^{2}} p(t) \sqrt{2 \pi} \frac{1}{2 \sqrt{1+t^{2}}} \\
& =\sqrt{2 \pi}\left[\frac{1}{\sqrt{2 \pi}} \int_{-T}^{T} e^{i s t} d \mu(s)\right]\left[\sqrt{2 \pi} \frac{1}{2 \sqrt{1+t^{2}}}\right]
\end{aligned}
$$

and applying the Fourier transform, we obtain, by (20),

$$
\int_{-T}^{T} K_{0}(y-v) d \mu(v)=1,|y| \leqslant T,
$$

with $K_{0}$ the Bessel function defined by 


$$
K_{0}(y)=\frac{1}{2} \int_{-\infty}^{\infty} \frac{e^{-i y v}}{\sqrt{1+v^{2}}} d v .
$$

It is not hard to see that $K_{0}(y)$ has the form $-\log y+b(y)$, with $b$ continuous, and for this case the existence of a solution $d \mu$ to (23) was asserted in [6]. As no proof was given, however, we supply a construction.

LEMMA 1. Suppose $K(t)$ (not identically constant) is nonnegative, even, integrable, and for $t>0$ is nonincreasing and convex. Then the equation

$$
\int_{-T}^{T} K(t-s) d \mu(s)=1 \text {, a.e., }|t| \leqslant T,
$$

has a unique solution which is a positive measure.

Remark. We expect that equality holds everywhere in (24) but we have no proof that the convolution is continuous.

Proof. The idea of the proof is to differentiate (24) so as to convert it into an equation of the second kind, which can be solved by iteration. Suppose first that $K^{\prime \prime}$ is continuous for $x \neq 0, K^{\prime \prime}(0+)<\infty, K^{\prime}(-T)>0$, and $K^{\prime}(0-)<\infty$; we will construct a solution of the form

$$
d \mu(s)=A\{\delta(s+T)+\delta(s-T)+f(s) d s\},
$$

with $f$ continuous. To this end, let

$$
M(x)=\frac{K^{\prime \prime}(x)}{2 K^{\prime}(0-)}, x \neq 0, M(0)=0
$$

and

$$
g(x)=\frac{K^{\prime \prime}(x-T)+K^{\prime \prime}(x+T)}{2 K^{\prime}(0-)},|x| \leqslant T
$$

Let $M$ denote the integral operator on $[-T, T]$ with kernel $M(x-y)$. We find, taking the discontinuity of $K^{\prime}$ at 0 into account, that

$$
|M h| \leqslant \sup |h| \int_{-T}^{T}|M(x-y)| d y
$$

and

$$
\begin{aligned}
\sup _{|x| \leqslant T} \int_{-T}^{T}|M(x-y)| d y & =\sup _{|x| \leqslant T} \int_{-T}^{x} M(x-y) d y+\int_{x}^{T} M(x-y) d y \\
& =\sup _{|x| \leqslant T}\left\{1-\frac{K^{\prime}(x-T)-K^{\prime}(x+T)}{2 K^{\prime}(0-)}\right\} \leqslant \gamma<1 .
\end{aligned}
$$

Consequently, $(I-M)^{-1}$ exists and is a bounded operator on the space of bounded continuous functions in $|x|<T$; indeed, setting

$$
f=(I-M)^{-1} g=g+M g+M^{2} g+\ldots
$$

we see that $f$ is positive, $|x| \leqslant T$. Now consider

$$
h(x)=\int_{-T}^{T} K(x-y) d \mu(y)
$$


with $d \mu$ as above. We find

$$
h^{\prime}(x)=A\left[K^{\prime}(x-T)+K^{\prime}(x+T)+\int_{-T}^{T} K^{\prime}(x-y) f(y) d y\right] .
$$

On differentiating again, remembering that $K^{\prime}(x)$ has a jump discontinuity at $x=0$ while $f$ is continuous, we obtain

$$
\begin{aligned}
h^{\prime \prime}(x) & =A\left[K^{\prime \prime}(x-T)+K^{\prime \prime}(x+T)-2 K^{\prime}(0-) f(x)+\int_{-T}^{T} K^{\prime \prime}(x-y) f(y) d y\right] \\
& =A 2 K^{\prime}(0)[g-f+M f]=0,
\end{aligned}
$$

by definition of $f$. But $h$ is even, so $h$ is a constant; the choice $A=\left[2 K(T)+\int_{-T}^{T} K(y) f(y) d y\right]^{-1}$ makes $h(x) \equiv 1$ and so produces a solution of (24).

We now remove the extraneous hypotheses by approximation. Thus assume only that $K(T)>0$. Then we can choose kernels $K_{n}$ to which the preceding argument applies, with $K_{n}(x) \uparrow K(x),|x| \leqslant T$. We find corresponding measures $\mu_{n}$ such that $K_{n} \mu_{n}=1, n \geqslant 1$. Writing

$$
(f, \mu)=\int_{-T}^{T} f(y) d \mu(y),
$$

we see from the monotonicity of the kernels that

$$
\left(1, \mu_{n}\right)=\left(K_{n+1} \mu_{n+1}, \mu_{n}\right)=\left(\mu_{n+1}, K_{n+1} \mu_{n}\right) \geqslant\left(\mu_{n+1}, K_{n} \mu_{n}\right)=\left(1, \mu_{n+1}\right),
$$

so that the total mass of $\mu_{n}$ decreases. By the Helly-Bray argument [4, p. 261] a subsequence converges weakly to some measure $d \mu$. It is no surprise that this limit is a solution. For when $n>m, K_{n} \geqslant K_{m}$, hence $1=K_{n} \mu_{n} \geqslant K_{m} \mu_{n}$, thus by weak convergence $1 \geqslant K_{m} \mu$, and letting $m \rightarrow \infty, 1 \geqslant K \mu$ by monotone convergence. Now choosing $\nu$ to be any measure for which $K \nu$ is continuous, we find

$$
(1, \nu)=\left(K_{n} \mu_{n}, \nu\right)=\left(K_{n} \nu, \mu_{n}\right) \leqslant\left(K \nu, \mu_{n}\right) \rightarrow(K \nu, \mu) \leqslant(\nu, 1)
$$

This shows that $K \mu=1$ a.e. For the final restriction, if $K(T)=0$, let $K_{\epsilon}=K+\epsilon, \epsilon>0$, and construct $\mu_{\epsilon}$ for which $K_{\epsilon} \mu_{\epsilon}=1$. Then $K \mu_{\epsilon}+\epsilon\left(1, \mu_{\epsilon}\right)=1$. If $\epsilon\left(1, \mu_{\epsilon}\right)=1$, then $K \mu_{\epsilon}=0$ and this, as we show next, implies that $\mu_{\epsilon} \equiv 0$, a contradiction. Thus we may take $\mu=\mu_{\epsilon} /\left[1-\epsilon\left(1, \mu_{\epsilon}\right)\right]$.

To prove that $\mu$ is unique we invoke the positive definiteness of $K$. Specifically, let us extend $K(t)$ into $|x|>T$ in any way which maintains the hypotheses, and so that $K(t)$ is a constant $\gamma \geqslant 0$ outside a compact set. We form the Fourier transform of $K_{1}(t)=K(t)-\gamma$, and integrate by parts twice to obtain

$$
\begin{aligned}
\hat{K}_{1}(s)=\int_{-\infty}^{\infty} K_{1}(t) e^{i t s} d t & =\frac{2}{s} \int_{0}^{\infty} K_{1}(t) d(\sin s t) \\
& =\frac{2}{s^{2}} \int_{0}^{\infty}(1-\cos s t) d K^{\prime}(t) \geqslant 0 .
\end{aligned}
$$

Consequently, 


$$
(K \mu, \mu)=\gamma|\hat{\mu}(0)|^{2}+\frac{1}{2 \pi} \int_{-\infty}^{\infty} \hat{K}_{1}(s)|\hat{\mu}(s)|^{2} d s \geqslant 0 .
$$

Since $K_{1}(t)$ and $d \mu$ vanish outside a compact set, their Fourier transforms are analytic functions of $s$ and if not identically zero can vanish at only a denumerable number of points. Thus if $(K \mu, \mu)=0$, necessarily $d \mu \equiv 0$. Applied to the difference of two solutions of (24), this argument establishes the uniqueness of $d \mu$. This completes the proof of Lemma 1.

For the second approach to (19) and (20), let us consider the space of functions $L^{2}(W)$ square-integrable with respect to the weight $W(x)=1 / 2 \sqrt{1+x^{2}}$. Thus we admit into the space functions like $(1+|x|)^{-1 / 2}$, which are not square-integrable. By the completeness of $L^{2}$, Cauchy sequences in this metric converge as well, so that $L^{2}(W)$ is a Hilbert space with scalar product

$$
[f, g]=\int_{-\infty}^{\infty} f(x) \overline{g(x)} \frac{d x}{2 \sqrt{1+x^{2}}} .
$$

Let us denote by $E_{T}$ the collection of functions which are the restrictions to the real axis of entire functions of the complex variable $(x+i y)$, having exponential type at most $T$, and by $H(T)$ the set of those functions in $E_{T}$ which are also in $L^{2}(W)$. Given $f(x) \in H(T)$, the function $f_{\epsilon}(x) \equiv f(x) \sin \epsilon x / \epsilon x$ is square-integrable for each $\epsilon>0$, since

$$
\begin{aligned}
\int_{-\infty}^{\infty}\left|f_{\epsilon}(x)\right|^{2} d x & =\int_{-\infty}^{\infty}|f(x)|^{2} \frac{1}{2 \sqrt{1+x^{2}}} 2 \sqrt{1+x^{2}} \frac{\sin ^{2} \epsilon x}{(\epsilon x)^{2}} d x \\
& \leqslant B \int_{-\infty}^{\infty}|f(x)|^{2} \frac{d x}{2 \sqrt{1+x^{2}}},
\end{aligned}
$$

with $B=\max _{x} 2 \sqrt{1+x^{2}} \sin ^{2} \epsilon x /(\epsilon x)^{2}<\infty$. Simultaneously, $f_{\epsilon}(x)$ extends to a function of exponential type at most $T+\epsilon$. Thus if $f_{n}(x) \epsilon H(T)$ form a Cauchy sequence converging to $f(x)$ in $L^{2}(W),\left\{f_{n, \epsilon}(x)\right\}$ is a Cauchy sequence in $L^{2}$, converging to $f(x) \sin \epsilon x / \epsilon x$. By the Paley-Wiener theorem, $f(x) \sin \epsilon x / \epsilon x$ is of exponential type at most $T+\epsilon$. On letting $\epsilon \rightarrow 0$, we see that $f(x)$ is of exponential type at most $T$, so that $H(T)$ is a closed subspace of $L^{2}(W)$, hence itself a Hilbert space.

LEMMa 2. There exists a unique solution to (19) and (20).

Proof. Given $f(x) \in H(T)$, we have seen that the corresponding $f_{\epsilon}(x) \epsilon L^{2} \cap E_{T+\epsilon}$, so by the Paley-Wiener theorem has Fourier transform supported in $|u| \leqslant T+\epsilon$. It follows by Schwarz's inequality and Parseval's theorem that

$$
\left|f_{\epsilon}(0)\right|^{2}=\left|\frac{1}{\sqrt{2 \pi}} \int_{-T-\epsilon}^{T+\epsilon} \hat{f}_{\epsilon}(u) d u\right|^{2} \leqslant \frac{1}{2 \pi} 2(T+\epsilon) \int_{-\infty}^{\infty}\left|f_{\epsilon}(x)\right|^{2} d x,
$$

and so, by (25),

$$
|f(0)|^{2}=\left|f_{\epsilon}(0)\right|^{2} \leqslant \frac{1}{\pi}(T+\epsilon) B \int_{-\infty}^{\infty}|f(x)|^{2} \frac{d x}{2 \sqrt{1+x^{2}}} .
$$

This asserts that the linear operation which maps $f(x) \in H(T)$ into $f(0)$ is a bounded linear functional in $H(T)$, hence by the Riesz representation theorem there exists a unique $h(x) \in H(T)$ such that 


$$
f(0)=[f, \bar{h}]=\int_{-\infty}^{\infty} f(x) h(x) \frac{d x}{2 \sqrt{1+x^{2}}}
$$

for each $f \in H(T)$. Now as $h \in H(T)$,

$$
p(x) \equiv h(x) / 2 \sqrt{1+x^{2}}
$$

is in $L^{2}$. If we choose $f \in H(T)$ also to be in $L^{2}$, and apply Parseval's theorem to (26), we obtain

$$
f(0)=\frac{1}{\sqrt{2 \pi}} \int_{-T}^{T} F(u) d u=\int_{-T}^{T} F(u) P(u) d u,
$$

with $P(u)$ the Fourier transform of $p(x)$. Since this must be true for each $F(u) \in L^{2}$, we conclude that $P(u)=1 / \sqrt{2 \pi}$ a.e., $|u| \leqslant T$, equivalently that (20) holds (a.e). Simultaneously, since $2 \sqrt{1+x^{2}} p(x)=h(x) \in H(T)$, (19) holds. Thus $p(x)$ is the required solution. The argument applies equally well to any weight $W(x)$ which decreases no faster than $t^{-n}$ for some $n$. This completes the proof of Lemma 2.

As we have seen, we can determine $u(x, y)$ from the solution of (19) and (20). However, less information is necessary to establish the asymptotic behavior (7) and (8).

Lemma 3. As $x \rightarrow-\infty$, the probability that the process hits $I_{L}$ is, asymptotically,

$$
Q(c, L, x) \sim \sqrt{\frac{\pi}{2 c|x|}}^{\mu}[-T, T],
$$

while the conditional probabilities that the process first hits $I_{L}$ on the near and far sides, given that it hits $I_{L}$, are, respectively,

$$
\frac{1}{2} \pm \frac{T}{\pi \mu[-T, T]}
$$

with $T=L c$ and $\mu$ the solution of (23).

Proof. We know that

$$
\begin{aligned}
& Q(c, L, x)=\operatorname{Re}+\operatorname{Im}\{u(x, 0)\}, \\
& R(c, L, x)=\operatorname{Im} u(x, 0) / Q(c, L, x) .
\end{aligned}
$$

Now when $x<0$

$$
\begin{aligned}
u(x, 0) & =\frac{1}{\sqrt{2 \pi}} \int_{-\infty}^{\infty} a(r) e^{\left(-c+\sqrt{c^{2}+r^{2}}\right) x} d r, \\
& =\frac{2}{\sqrt{2 \pi}} \int_{0}^{\infty} a(r) e^{\left(-c+\sqrt{c^{2}+r^{2}}\right) x} d r, \\
& =\frac{2}{\sqrt{2|x|}} \int_{0}^{\infty} a\left(\sqrt{s^{2}+2 c s}\right) e^{-s|x|} \sqrt{\frac{|x|}{\pi}} \frac{d s}{\sqrt{s}} \frac{s+c}{\sqrt{s+2 c}},
\end{aligned}
$$

by the evenness of $a$ and the substitution $s=\sqrt{c^{2}+r^{2}}-c$. As the kernel $\sqrt{|x| / \pi} e^{-s|x|} / \sqrt{s}$ approaches $\delta(0)$ when $|x| \rightarrow \infty$, 


$$
u(x, 0) \sim \sqrt{\frac{c}{|x|}} a(0) .
$$

But from (19), (21), and (22),

$$
\begin{aligned}
a(0) & =\frac{1}{2}\left[(1-i) \frac{2 L}{\sqrt{2 \pi}}+\frac{\sqrt{2 \pi}(1+i)}{c} p(0)\right] \\
& =\frac{1}{2}\left\{(1-i) \frac{2 L}{\sqrt{2 \pi}}+\frac{\sqrt{2 \pi}(1+i)}{2 c} \mu[-T, T]\right\},
\end{aligned}
$$

and Lemma 3 now follows from (27).

In returning to the measure $\mu$, we should note that the study of Hilbert spaces of functions of exponential type, square-integrable on the reals with respect to a weight, is of interest in its own right [2]. In that context, the determination of the evaluation element (or of $\mu[-T, T]$ which measures its norm) from the integral equation which corresponds to (23) is a central problem. A broad theory of such equations, which allows efficient construction of solutions, has recently been developed [7]. Here, however, we will restrict ourselves to (23) and treat it by a Wiener-Hopf method.

THEOREM. Let $d \mu(x)$ be the solution of

$$
\int_{-T}^{T} K_{0}(y-x) d \mu(x)=1,|y| \leqslant T .
$$

Then $\mu[-T, T]=(1+2 T) / \pi+o\left(e^{-2 T}\right)$. For small $T, \mu[-T, T] \sim-1 / \log T$.

Proof. The equation has the general character of a Wiener-Hopf problem, in which the convolution is prescribed only over the support of the unknown function. Accordingly, we will treat it by extending the convolution to $|y|>T$ and, using projections, reason about the (unknown) values thus introduced.

To begin with, let us recall that, as in (22), on setting

$$
\begin{aligned}
& h(t)=\int_{-T}^{T} e^{i s t} d \mu(s), \\
& p(t)=\frac{h(t)}{2 \sqrt{1+t^{2}}},
\end{aligned}
$$

equation (28) is equivalent, under Fourier transformation, to asserting that $P(u)=1 / \sqrt{2 \pi},|u| \leqslant T$. Now $p(t) \in L^{2}$, so $P$, likewise in $L^{2}$, can be decomposed into three mutually orthogonal components obtained by restricting $P$ to the intervals $(-\infty,-T),[-T, T]$, and $(T, \infty)$, respectively. On writing $p$ as the inverse transform of $P$, we obtain correspondingly

$$
p(t)=q^{-}(t)+\frac{1}{\sqrt{2 \pi}}\left(\frac{1}{\sqrt{2 \pi}} \frac{e^{i T t}-e^{-i T t}}{i t}\right)+q^{+}(t),
$$

in which the components remain mutually orthogonal (the Fourier transform being unitary in $L^{2}$ ), and the middle one is determined by (20). Thus

$$
\int_{-\infty}^{\infty}|p(t)|^{2} d t=\int_{-\infty}^{\infty}\left|q^{-}(t)\right|^{2} d t+\int_{-\infty}^{\infty}\left|q^{+}(t)\right|^{2} d t+\frac{1}{2 \pi}(2 T) .
$$


We need a bound for the integrals on the right-hand side of (30); we can assume them equal by symmetry. For this purpose we observe that, as in Lemma $2, h(t)$ is the representing element for evaluation at $t=0$ in the Hilbert space $H(T)$. From (26)

$$
h(0)=[h, h]=\int_{-\infty}^{\infty} \frac{|h(t)|^{2}}{2 \sqrt{1+t^{2}}} d t,
$$

and since this quantity is the square of the norm of the linear functional, we have equivalently

$$
\int_{-\infty}^{\infty} \frac{|h(t)|^{2}}{2 \sqrt{1+t^{2}}} d t=\sup _{f \in E_{T}} \frac{|f(0)|^{2}}{\int_{-\infty}^{\infty}|f(t)|^{2} / 2 \sqrt{1+t^{2}} d t}
$$

Since

$$
\begin{gathered}
\int_{-\infty}^{\infty}|f(t)|^{2} / 2 \sqrt{1+t^{2}} d t \geqslant \int_{-\infty}^{\infty}|f(t)|^{2} / 2\left(1+t^{2}\right) d t \\
\sup _{f \in E_{T}} \frac{|f(0)|^{2}}{\int_{-\infty}^{\infty}|f(t)|^{2} / 2 \sqrt{1+t^{2}} d t} \leqslant \sup _{f \in E_{T}} \frac{|f(0)|^{2}}{\int_{-\infty}^{\infty}|f(t)|^{2} / 2\left(1+t^{2}\right) d t} .
\end{gathered}
$$

The last quantity can be viewed as the square of the norm of the functional $f \rightarrow f(0)$ in the Hilbert space of functions $f \in E_{T}$, with the norm given by $\left[\int|f(t)|^{2} / 2\left(1+t^{2}\right) d t\right]^{1 / 2}$. By the argument of Lemma 2, this equals $q(0)$, where $q \in E_{T}$ and the Fourier transform of $q(t) / 2\left(1+t^{2}\right)$ coincides with $1 / \sqrt{2 \pi}$ for $|u| \leqslant T$. Since $1 / 2\left(1+t^{2}\right)$ is the Fourier transform of $(\sqrt{2 \pi} / 4) e^{-|x|}$, this latter requirement is in turn equivalent to

$$
\int_{-T}^{T} \frac{\pi}{2} e^{-|x-y|} d \nu(x)=1,|y| \leqslant T,
$$

with

$$
q(t)=\int_{-T}^{T} e^{i t x} d \nu(x) .
$$

This is useful because (34) can be solved explicitly, to give

$$
d \nu(x)=\frac{1}{\pi}[\delta(T)+\delta(-T)+1],|x| \leqslant T,
$$

so that from (35)

$$
q(0)=\frac{1}{\pi}(2+2 T)
$$

On combining (31),(33), and (36) we find

$$
\int_{-\infty}^{\infty} \frac{|h(t)|^{2}}{2 \sqrt{1+t^{2}}} d t \leqslant \frac{1}{\pi}(2+2 T),
$$

and since by (32) 


$$
\int_{-\infty}^{\infty}|p(t)|^{2} d t=\int_{-\infty}^{\infty} \frac{|h(t)|^{2}}{4\left(1+t^{2}\right)} d t \leqslant \frac{1}{2} \int_{-\infty}^{\infty} \frac{|h(t)|^{2}}{2 \sqrt{1+t^{2}}} d t,
$$

we obtain finally

$$
\int_{-\infty}^{\infty}|p(t)|^{2} d t \leqslant \frac{1}{2 \pi}(2+2 T)
$$

Applied to (30), this bound shows that

$$
\int_{-\infty}^{\infty}\left|q^{ \pm}(t)\right|^{2} d t \leqslant \frac{1}{2 \pi}
$$

Next, let us return to $(28)$, which we replace by

$$
\int_{-T}^{T} K_{0}(y-x) d \mu_{\epsilon}(x)=e^{-\epsilon y},|y| \in T,
$$

with $\epsilon>0$. The solution exists by the argument of Lemma 2 applied to evaluation at $-i \epsilon$ instead of at 0 . This is done in order to modify the decomposition (29) to

$$
\frac{h_{\epsilon}(t)}{2 \sqrt{1+t^{2}}}=q_{\epsilon}^{-}(t)+\frac{1}{\sqrt{2 \pi}}\left(\frac{1}{\sqrt{2 \pi}} \frac{e^{-(\epsilon+i t) T}-e^{(\epsilon+i t) T}}{-\epsilon-i t}\right)+q_{\epsilon}^{+}(t),
$$

for moving the zero of the denominator of the middle term away from the real axis will enable us to obtain asymptotics by the technique of Wiener-Hopf equations on a half-line. We are grateful to the referee for pointing out that this approach can be motivated physically in the following way. Let us make $T=L c$ large by increasing the drift, and picture the diffusion as starting a little above the negative real axis, at $y=\epsilon$; then in order to clear the left side of the segment $|y|<L$, the particle requires an upward displacement exceeding $(L-\epsilon)$ or a downward one exceeding $(L+\epsilon)$ before reaching the segment. As the magnitude of the horizontal drift increases, the time allowed for this is progressively shorter, and the latter event becomes increasingly unlikely by comparison with the former. Thus, in effect, the segment can be extended to $y \leqslant L$ without affecting the probability of interest, and the problem becomes posed on a half-line.

Clearly, as $\epsilon \rightarrow 0, d \mu_{\epsilon} \rightarrow d \mu$ and for the quantity of interest we have from (38)

$$
\int_{-T}^{T} d \mu(s)=\lim _{\epsilon \rightarrow 0} \int_{-T}^{T} d \mu_{\epsilon}(s)=\lim _{\epsilon \rightarrow 0} h_{\epsilon}(0)=\lim _{\epsilon \rightarrow 0} 2\left[q_{\epsilon}^{-}(0)+q_{\epsilon}^{+}(0)\right]+\frac{2 T}{\pi} .
$$

We now multiply (38) by $e^{i t T} \sqrt{t-i}$ to obtain

$$
e^{i T t} \frac{h_{\epsilon}(t)}{2 \sqrt{t+i}}=r_{-}(t)+r_{+}(t),
$$

with

$$
\begin{aligned}
& r_{-}(t)=q_{\epsilon}^{-}(t) e^{i T t} \sqrt{t-i}-\frac{1}{2 \pi} \frac{e^{-\epsilon T}}{\epsilon+i t} \sqrt{t-i}, \\
& r_{+}(t)=q_{\epsilon}^{+}(t) e^{i T t} \sqrt{t-i}+\frac{1}{2 \pi} \frac{e^{\epsilon T} e^{2 i T t}}{\epsilon+i t} \sqrt{t-i} .
\end{aligned}
$$

The left-hand side of (40) remains square-integrable, since $h_{\epsilon}(t) \in H(T)$ and we will 
assume that likewise $r_{ \pm}(t) \in L^{2}$. Since $h_{\epsilon}(t)$ can be extended into the complex $(t+i u)$ plane to be an entire function of exponential type not exceeding $T$, $e^{i t T} h_{\epsilon}(t) / \sqrt{t+i}$ is extendable to be analytic and bounded in the upper half-plane and so by the Paley-Wiener theorem it is a function of $\mathrm{H}_{2}^{+}$, i.e. one representable as $\int_{0}^{\infty} H(s) e^{i t s} d s$, with $H \in L^{2}$. Analogously, since by definition $q_{\epsilon}^{-}(t)=\int_{-\infty}^{-T} Q_{\epsilon}(s) e^{i t s} d s$, the frequencies of $q_{\epsilon}^{-}(t)$ are contained in $s<-T$, so $r_{-}(t)$ is extendable analytically to the lower half-plane, hence is contained in the complementary subspace $\mathrm{H}_{2}^{-}$. Thus projecting (40) onto $\mathrm{H}_{2}^{-}$we find

$$
0=r_{-}(t)+\mathbf{P}\left[r_{+}(t)\right],
$$

where $\mathbf{P}$, the projection in $L^{2}$ onto $H_{2}^{-}$, is given explicitly by

$$
(\mathbf{P} f)(t)=\lim _{\substack{z \rightarrow t \\ \operatorname{Im} z<0}}-\frac{1}{2 \pi i} \int_{-\infty}^{\infty} \frac{f(u)}{u-z} d u \text {. }
$$

The assumption that $r_{ \pm} \in L^{2}$ represents no loss of generality, for we can ensure it by multiplying (40) by the convergence factor $\left(1-e^{-i \delta t}\right) / i \delta t$; as the left-hand side of $(40)$ so modified converges to $\mathrm{H}_{2}^{+}$when $\delta \rightarrow 0$, we obtain (41) in the limit. To estimate $\mathbf{P} \boldsymbol{r}_{+}$we deform the contour of integration into the upper half-plane. Specifically, let $\boldsymbol{\Gamma}$ denote the boundary of the semidisk of radius $N$ in the upper half of the $\zeta=u+i v$ plane, from which the vertical slit $1 \leqslant v \leqslant N$ has been removed. Then $\Gamma$ consists of: $|u| \leqslant N ;|\zeta|=N, 0<\arg \zeta<\pi / 2$; the slit $u=0,1 \leqslant v \leqslant N$ traversed twice; $|\zeta|=N, \pi / 2<\arg \zeta<\pi$. Now in the region bounded by $\Gamma, \sqrt{\zeta-i}$ is single-valued, and the second component of $r_{+}$has a simple pole at $\zeta=i \epsilon$. Consequently, for $\operatorname{Im} z<0$,

$$
\frac{1}{2 \pi i} \int_{-N}^{N} \frac{r_{+}(u)}{u-z} d u=\frac{1}{2 \pi i} \int_{\Gamma}-\frac{1}{2 \pi i} \int_{|\zeta|-N}-\frac{1}{2 \pi i} \int_{\text {slit }} \frac{r_{+}(\zeta)}{\zeta-z} d \zeta
$$

The first of these components is given by the residue of $r_{+}(\zeta) /(\zeta-z)$ at $\zeta=i \epsilon$,

$$
\frac{1}{2 \pi i} \int_{\Gamma} \frac{r_{+}(\zeta)}{\zeta-z} d \zeta=\frac{1}{i} \frac{1}{2 \pi} \frac{e^{-\epsilon T} \sqrt{i \epsilon-i}}{i \epsilon-z}
$$

For the second, we use the definition

$$
q_{\epsilon}^{+}(t)=\int_{T}^{\infty} Q(s) e^{i s t} d s
$$

with some $Q(s) \in L^{2}$, to see that $e^{i T \zeta} q_{\epsilon}^{+}(\zeta)=O\left(e^{-2 T \nu}\right)$ in the upper half-plane; thus the second component of (43) approaches 0 as $N \rightarrow \infty$. We conclude from (41), (42), and (43) that

$$
-\frac{1}{2 \pi} \frac{e^{-\epsilon T}}{\epsilon+i t} \sqrt{t-i}+q_{\epsilon}^{-}(t) e^{i T t} \sqrt{t-i}=\lim _{\substack{z \rightarrow t \\ \operatorname{Im} z<0}} \frac{1}{i} \frac{1}{2 \pi} \frac{e^{-\epsilon T} \sqrt{i \epsilon-i}}{i \epsilon-z}-\frac{1}{2 \pi i} \int_{\text {slit }} \frac{r_{+}(i v)}{i v-z} i d v \text {. }
$$

Letting $t=0$ in (45) we find 


$$
\begin{aligned}
q_{\epsilon}^{-}(0) & =\frac{1}{2 \pi} \frac{e^{-\epsilon T}}{\epsilon}+\frac{1}{2 \pi} \frac{1}{i \sqrt{-i}} \frac{e^{-\epsilon T} \sqrt{i \epsilon-i}}{i \epsilon}-\frac{1}{2 \pi \sqrt{-i}} \int_{\text {slit }} \frac{r_{+}(i v)}{i v} d v \\
& =\frac{1}{2 \pi} \frac{e^{-\epsilon T}}{\epsilon}[1-\sqrt{1-\epsilon}]-\frac{1}{2 \pi i \sqrt{-i}} \int_{\text {slit }} \frac{r_{+}(i v)}{v} d v .
\end{aligned}
$$

Now the slit is given by $v \geqslant 1$, and is traversed twice. For an estimate of $r_{+}(i v)$, we see from (44) that $2 \pi \int_{T}^{\infty}|Q(s)|^{2} d s=\int_{-\infty}^{\infty}\left|q_{\epsilon}^{+}(t)\right|^{2} d t$, while by (37) the latter quantity is bounded by a constant. Then by Schwarz's inequality, $\left|q_{\epsilon}^{+}(i v)\right| \leqslant c e^{-T v} / \sqrt{v}$, so that

$$
\left|r_{+}(i v)\right| \leqslant c \frac{e^{-2 T v}}{\sqrt{v}} .
$$

Finally, combining (39), (46), and (47), and using symmetry to conclude that $\lim _{\epsilon \rightarrow 0} q_{\epsilon}^{-}(0)=\lim _{\epsilon \rightarrow 0} q_{\epsilon}^{+}(0)$, we obtain

$$
\int_{-T}^{T} d \mu(s)=\frac{1+2 T}{\pi}+o\left(e^{-2 T}\right) .
$$

When $T$ is small, we appeal to [4], where the solution of (27) with the kernel $\log (1 /|y|)$ is given explicitly as

$$
d \mu(x)=\frac{1}{\log \frac{2}{T}} \frac{1}{\pi} \frac{1}{\sqrt{T^{2}-s^{2}}} d s, \quad T<1 / 2,
$$

so that $\int_{-T}^{T} d \mu(x)=1 / \log (2 / T)$. Since $K_{0}(y) \sim \log (1 /|y|)$ as $T \rightarrow 0$, the remaining estimate of the Theorem follows.

\section{REFERENCES}

[1] H. C. BERG and E. M. PURCELL, Physics of chemoreception, Biophysics J., 20 (1977), pp. 193-219.

[2] L. DeBranges, Hilbert Spaces of Entire Functions, Prentice-Hall, New York, 1968.

[3] D. W. STROOCK and S. R. S. varAdHAN, Multidimensional Diffusion Processes, Springer-Verlag, Berlin, 1979.

[4] W. FELLER, An Introduction to Probability Theory and Its Applications II, Wiley, New York, 1966, 1st ed.

[5] M. G. KREIN, On a method for effectively solving an inverse boundary value problem, Doklady Akad. Nauk SSSR, 94 (1954), pp. 987-990.

[6] M. G. KREIN, On a new method of solving linear integral equations of the first and second kind, Doklady Akad Nauk SSSR, 100 (1955), pp. 413-416.

[7] B. F. LOGAN, to appear.

[8] R. M. MACNAB and D. E. KOSHLAND, JR., The gradient-sensing mechanism in bacterial chemotaxis, Proc. Nat. Acad. Sci. 69 (1972), pp. 2509-2512.

[9] S. H. ZIGMOND, Ability of polymorphonuclear leukocytes to orient in gradients of chemotactic factors, J. Cell Biology, 75 (1977), pp. 606-616. 